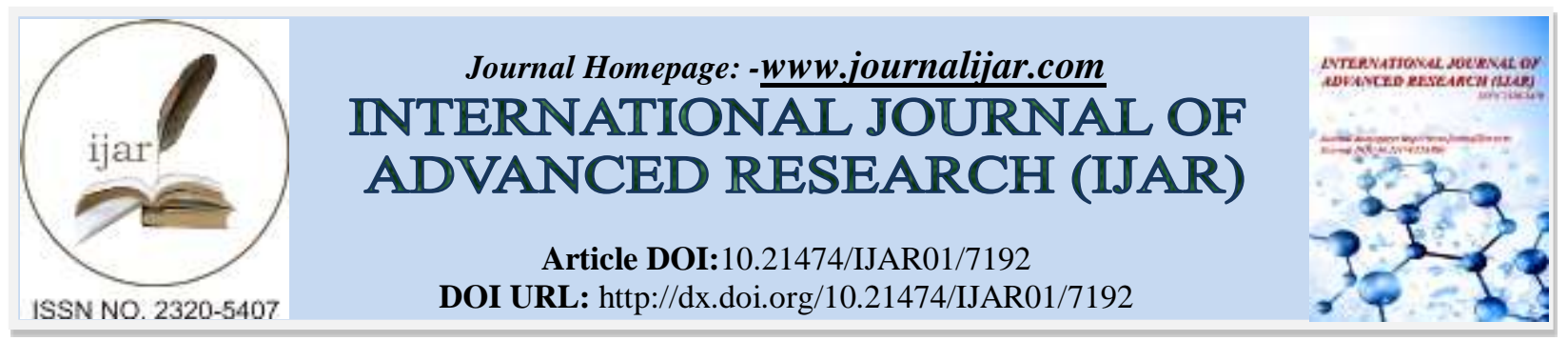

RESEARCH ARTICLE

\title{
FINANCEMENT DES PME - ETUDE QUANTITATIVE SUR LES BANQUES LIBANAISES.
}

Prof. Kamel barbar and Fatima Roumani.

1. Doyen du Business School Jinan University - Liban.

2. Doctorant en sciences de gestion.

\section{Manuscript Info}

Manuscript History

Received: 03 April 2018

Final Accepted: 05 May 2018

Published: June 2018

\section{Abstract}

Les recherches faites auparavant sur les PME affirment que celles-ci souffrent d'un obstacle d'accès au financement. Cet obstacle se manifeste à deux niveaux. Le premier serait le coût du financement bancaire du fait que ce type d'entreprises se voit offert des taux d'intérêt plus élevé que les grandes entreprises et des conditions d'octroi bien chères comme les garanties exigées. Le deuxième niveau serait la durée de l'emprunt. En effet, les PME trouvent une difficulté de s'acquérir un financement au long terme qui est la meilleure façon d'emprunter pour assurer une stabilité de financement et une meilleure répartition des coûts financiers.

Cette étude cherche à investiguer sur la situation actuelle du financement des PME libanaises par emprunt bancaire. En d'autres termes, il s'agit de s'informer et de mettre la lumière sur la facilité ou la difficulté au niveau de l'accès des PME libanaises au crédit bancaire.

L'investigation sera faite par une enquête auprès d'un échantillon de chargés d'affaires au sein de banques libanaises. Les résultats mettront en évidence les obstacles rencontrés par les PME libanaises cherchant à se financer auprès des banques et les facteurs décisifs à l'octroi des crédits bancaires.

Les résultats de l'enquête mettent en relief les méthodes d'analyse des demandes de financement des PME au sein du secteur bancaire. L'analyse reflète les facteurs qui influencent la décision d'octroi ou de refus du crédit. Parmi ces facteurs on cite : l'historique du client, son expérience, l'étude de faisabilité, le secteur d'activité et surtout les garanties disponibles.

La situation actuelle qui se traduit par une difficulté d'accès au crédit bancaire ou plutôt à des conditions de crédit plus adéquates requiert un remède. Ce remède est sans doute une meilleure transparence de la part des PME et la disponibilité d'information nécessaire à l'octroi de crédit. De sa part, le secteur bancaire doit revoir sa politique de crédit envers les PME et faciliter les conditions d'octroi.

Copy Right, IJAR, 2018,. All rights reserved. 


\section{Introduction:-}

Aperçu Général:-

Les travaux étudiant spécifiquement la population PME soutiennent l'existence d'un «écart permanent dans les conditions d'accès au financement de ces entreprises». Un écart dans les conditions d'accès au financement est en général défini par deux caractéristiques (Garvin, 1971). Premièrement, le coût d'accès au financement est plus élevé pour les petites entreprises. Cette caractéristique a été soulignée sur le cas français et en ce qui concerne le marché du crédit par Bardos (1991). Deuxièmement, les PME «souffriraient» d'un déficit chronique de financement de longue période, que ce soit par émission d'actions ou par emprunts bancaires. Cette caractéristique a comme corollaire un endettement à court terme important qui peut expliquer la plus forte sensibilité de ces entreprises aux chocs conjoncturels. L'endettement étant, par ailleurs, la principale source de financement, le gonflement des besoins en fonds de roulement et ou la chute du chiffre d'affaires ne peuvent pas être compensés, comme chez les grandes entreprises, par un recours accru au financement externe. Ces caractéristiques conduisent certains économistes ou décideurs politiques à soutenir l'hypothèse de l'existence d'un système financier discriminatoire à l'égard des petites entreprises et à justifier l'existence d'institutions financières spécialisées dans le financement de cette catégorie d'entreprises.

\section{Objectif de l'Etude:-}

La proximité et la confiance sans oublier qu'une bonne gestion de l'information concernant l'obtention de crédit peut réduire le risque et l'incertitude. Les débats sont nombreux au Liban concernant l'importance de l'obstacle financier à la création et au développement de la P.M.E. Les pouvoirs publics considèrent ce type d'entreprise comme un instrument de relance de l'économie nationale mais les banquiers sont réticents à donner les crédits nécessaires vu les risques et incertitudes de cette catégorie d'entreprise. C'est ainsi que chaque fois qu'une P.M.E demande que emprunt bancaire, elle se voit soumise à l'obligation de présenter d'énormes garantis qu'elle ne peut satisfaire et c'est ainsi qu'elle se trouve écarté du crédit bancaire. Bien que les travaux de Hugon (1990) montrent que le financement informel est limité par la taille des dépôts et ne peut permettre de financier l'investissement.

Certaines questions doivent être posées :

1. Doit-on continuer à abandonner ce type d'entreprise à elle même face à la méfiance des banques à financer la P.M.E.?

2. Tous les projets des P.M.E refusés par les banques ne sont pas valables?

3. Quel serait le coût social des dossiers refusés en termes d'emplois?

4. Est-ce que les banques doivent avoir une capacité à collecter et à produire de l'information relative aux réputations et aux comportements des PME?

L'acquisition de l'information ou une information supplémentaire sur les avantages du projet ainsi que l'évaluation du coût de cette information deviennent des questions centrales. Donc compte tenu du poids économique des P.M.E au Liban nous allons essayer de trouver un terrain d'entente entre elles et les banques au lieu de les exclure du champ de crédit.

Comment peut-on y arriver ? Par une saisie de la qualité de l'information, principale condition à la constitution d'un réseau de confiance entre les banques et les P.M.E. Dans une première étape de notre étude, nous définirons l'incertitude, l'ambiguité et l'opacité au niveau de l'information chez les PME. Dans la deuxième étape nous expliquons les différents types d'asymétrie de l'information ainsi que le risque et ses sources. Alors que dans la troisième étape nous tentons de traiter le lien entre l'accroissement, la taille d'une banque et leur incidence sur l'accord des crédits aux P.M.E. Ensuite nous abordons le sujet de la source de l'information et son rôle dans l'octroi du crédit bancaire et finalement nous traitons la question de la communication de l'information et le risque du crédit. Cette démarche sera sur une adaptation des traditionnelles appréciations du risque en matière de l'obtention de crédit à la réalité de la P.M.E Libanaise et surtout prendre en compte les valeurs socioculturelles locales et la bonne gestion de l'information car elle peut entraîner des coûts énormes à l'entreprise.

\section{Revue de Littérature:-}

\section{Incertitude, Ambiguïté et Opacité dans la Communication des PME au Liban:-}

Dans le courant post Kéynesien, l'incertitude et le risque sont nombreux. Cette question à surtout travaillé le courant néo classique et les post- Kéynesiens vont essayer de différencier l'incertitude du risque. Le point de départ sera l'étude de la traite de la probabilité de Keynes (1921) et ses premiers écrits. Tous ces travaux seront axés sur une perception philosophique de l'incertitude qui est intrinsèquement liée à la connaissance. Pour Keynes, le futur dépend de l'inconnu et ne peut par conséquent être l'objet de calcul probabiliste. D'après lui, l'incertitude matérialise 
l'ignorance de la connaissance face au devenir car à long terme, nous serons tous morts. Davidson se fera le porte parole de cette théorie de l'acceptation des limites de la pensée rationnelle face au futur. Keynes justifie cette approche dans "le traité sur la probabilité" en distinguant la mesure dans les sciences morales et la mesure dans les sciences de la nature. Cette conviction l'amène dans ses écrits à insister sur le caractère précaire des méthodes de calcul du rendement escompté et c'est ainsi que son analyse permet d'affirmer que la rationalité est obligatoirement limitée et ce qui nous amène à le considérer comme précurseur de la rationalité développée par Simon (1982) et Quinet (1994). Le résultat sera que le traitement de l'information chez Simon ne sera pas indépendant du choix du décideur ainsi que le poids qui selon Keynes, ne pourra être expliqué en terme de probabilité. La connaissance économique est asymétrique: bien qu'il soit possible d'accepter le passé présent, nous ne pouvons produire l'avenir.

En définitive on peut résumer que le risque ne peut être étudié uniquement à travers les variables quantitatives car cela implique également la prise en compte des variables qualitatives. Nous pouvons dire en conclusion que le risque dépend de la probabilité objective, mais aussi du capital confiance et par conséquent du jugement relatif à la réputation. Nous rejoignons là Gambetta (1988) qui dans ses enseignements nous fait savoir que la confiance est nécessaire dès que les hypothèses d'information parfaite ne fonctionnent plus. Avant d'examiner les nouvelles relations financières entre les banques et les P.M.E Libanaises, étudions d'abord les sources du risque.

La difficulté majeure qui heurte les banques Libanaises réside dans leur incapacité de cerner le risque présenté par la P.M.E et cela les amène à fixer un niveau de risque élève à tout les prêt accordés à une P.M E. La surestimation du risque les fait exiger des primes trop élevées et par là, les clients se sentent éliminés d'office. Dans les deux cas, le résultat est le même: pas de crédit. L'excès de risque dans la P.M.E Libanaises a plusieurs origines: L'environnement, la gestion dans les P.M.E, l'absence d'information valable. Avant de rentrer dans le vif du sujet. Nous allons essayer de définir la P.M.E dans le contexte Libanais.

La définition de la P.M.E varie en fonction de l'objet de l'étude et des données disponibles. Le rapport Bolton retreint trois critères qualitatifs pour la définition d'une P.M.E: elle doit retenir une part plus ou moins petite de son marché, elle doit être gérée d'une manière personnelle par son propriétaire et elle doit être indépendante non intégrée dans une structure plus grande et ses propriétaire doivent prendre leurs décisions sans contrôle extérieur.

Cependant même lorsque le critère du nombre des salaires est retenu, des différences considérables persistent dans les pays et dans le cadre de ce travail nous serons amenés à distinguer la situation dans les trois catégories de P.M.E: la petite avec ses 10 employés, la moyenne avec ses 25 et la grande avec plus de 50 employés. Au delà de l'effectif du personnel il faut souligner des traits communs à ces entreprises.

\section{Perception des Sources de l'Ambiguïté Informationnelle:-}

La plupart des P.M.E n'ont donc pour véritable marché que le Liban et c'est aussi qu'il est difficile dans ces conditions pour la P.M.E de bénéficier des économies d'échelle relatives à une production de masse. La gestion: dans un contexte instable, la rigueur de gestion s'impose aux entreprises si elles veulent se maintenir sur le tissu économique. La surveillance de la gestion devient une exigence particulière. Si les grandes entreprises locales peuvent s'assurer les services de cadre et de spécialistes de haut niveau, le patron de la P.M.E au Liban est un individu, il doit faire face en même temps aux problèmes de politique générale et aux questions opérationnelles. Ses décisions sont le plus souvent prises à partir des données très insuffisantes et il faut relever le poids des contraints sur la gestion de l'entreprise qui ne sont pas toujours compatibles avec les règles les plus élémentaires du management, le gain spéculatif la confusion du patrimoine de l'entreprise avec le patrimoine domestique tout cela n'aide pas à rassurer le prêteur rationnel.

\section{L'imperfection de l'Information:-}

L'une des caractéristiques définissant la P.M.E est la simplicité de son système d'information externe: Ce système est basé sur des relations informelles que le dirigeant avec son environnement. La transmission de l'information est le plus souvent directe orale et peu formalisée. L'insuffisance de l'information peut être à un double niveau: sur la situation générale de l'entreprise et sur le plan comptable.

En générale au Liban, il existe un manque de connaissance des moyens de l'entreprise tant du côté humains ou matériel. On n'arrive pas à savoir la substance du patrimoine de l'entreprise et à délimiter les conteurs du patrimoine du chef. Les ponts constituent un élément important pour l'étude d'un dossier de demande de crédit. Ensuite, la P.M.E Libanaise se caractérise par la production d'informations comptable très ambiguës. A cet effet, on distingue 
un bilan fiscal négatif et un bilan bancaire positif. La santé réelle de l'entreprise en souffre de même que la relation banque- P.M.E et c'est ainsi que nous avons un informationnel et une attitude frileuse des banques à l'égard des P.M.E.

Au regard de l'importance économique de la P.M.E dans le tissu économique Libanais, nous devons nous orienter sur la problématique et l'étude du risque adapté au contexte de la P.M.E au Liban. L'hypothèse faite repose sur les possibilités des banques à aller au-delà des procédures actuelles d'appréciation des demandes de crédit formulée par les P.M.E et devenir enfin un véritable partenaire financier de ces dernières. La démarche consistera d'abord à dégager les spécificités du risque de la P.M.E Libanaise et ensuite déterminer les instruments d'analyse les plus aptes à répondre à ces spécificités viennent de l'étroitesse des marchés, de la conjoncture défavorables et instable, de la contrainte familiale pesant sur la P.M.E., le recrutement d'un personnel souvent incompétent et enfin l'absence de planification. Il s'avère que la planification des opérations dans une entreprise est des plus nécessaires car elle permet de formaliser les différentes éventuelles et de contrôler les résultats.

\section{Méthodologie de la Recherche:- \\ Echantillonnage:-}

Tout travail qui se dit scientifique doit obéir à un certain nombre de règles et de méthodes. Le choix d'un positionnement méthodologique est une étape importante dans le déroulement d'une recherche puisqu'il conditionne la production des connaissances. Selon Thiétart et al (2003) : "C'est à partir de connaissances fines des éléments qui composent un objet que le chercheur pourra tenter de comprendre les liens causaux qui se nouent entre ces éléments et qui expliquent finalement la forme de l'objet étudié ». Dans une première section, nous allons présenter notre méthodologie de recherche et le cheminement que nous allons adopter pour l'étude du phénomène en question. Il existe une vaste espace pour le positionnement méthodologique du chercheur. Cette variété des approches possibles est souhaitable en ce qu'elle recèle comme source d'enrichissements. Une recherche peut ainsi :

1. Se situer dans une logique inductive ou déductive.

2. Etre de type quantitatif ou qualitatif.

La distinction déductive et inductive fait référence à la relation entre la théorie et les observations empiriques. La connaissance produite peut être le fruit d'observations empiriques (démarche inductive) ou, au contraire, la connaissance théorique peut précéder les observations empiriques qui servent alors de vérification à la connaissance initiale (démarche déductive). La connaissance peut encore se construire par interaction entre la théorie et les données empiriques (démarche adductive).

Autrement dit la déduction est l'expression courante du positivisme (qu'on expliquera dans ce qui suit), allant du général au particulier.

Sur ce premier point, notre recherche reposera sur une démarche déductive, dans laquelle la collecte des données sera de type quantitatif à travers des questionnaires auprès des banques commerciales au Liban.

\section{Collecte d'Information:-}

«L'art d'interroger n'est pas si facile qu'on pense. C'est bien plus l'art des maitres que des disciples, il faut avoir déjà beaucoup appris de choses pour savoir demander ce qu'on ne sait pas » (J.J.Rousseau, in Boss.J.F). Elle nous permettra de répondre à notre problématique et à nos questions de recherche. "Le questionnaire n'est pas une simple série de questions. C'est un instrument de mesure à construire de façon logique et cohérente en veillant à mettre à l'aise et à intéresser la personne interrogée. L'ordre des questions (ou dynamique du questionnaire) joue un rôle essentiel.

Un questionnaire est une phase d'une enquête durant laquelle il est soumis pour être complété aux individus faisant partie de l'échantillon. Ce travail consiste à :

1. Choisir un échantillon de personnes à questionner.

2. Définir le type de questions à poser: questions fermées, ouvertes...

3. Structurer le questionnaire.

4. Déterminer l'endroit où doit se dérouler l'enquête (dans la rue, par courrier, sur Internet, ...).

5. Réaliser l'enquête: choix de la date, des interviewers, etc.

6. Organiser la saisie et traiter les résultats : tris à plat, tris croisés, moyennes. 
Dans le questionnaire, l'individu répond le fait dans un cadre fixé à l'avance par le spécialiste.

Le questionnaire a pour ambition première de saisir le sens objectif des conduites en les croisant avec des indicateurs des déterminants sociaux.

Pour rédiger un questionnaire, il faut dans un premier temps expliciter l'objectif principal de la recherche. Ensuite, définir les sous objectifs, les dimensions, les informations et enfin les questions à poser. C'est une démarche basée sur la logique de l' «entonnoir », c'est-à-dire du général au spécifique.

Nous sommes allés donc vers une connaissance scientifique basée sur la logique suivante: Lois \& Théories $\rightarrow$ Déductions $\rightarrow$ Explication \& Prévisions

\section{Analyse des Données:-}

L'épistémologie est : «la partie de la philosophie qui étudie les méthodes et les perspectives des sciences ». Nous nous devons de donner une description aussi fidèle de la réalité et c'est en ce sens que le problème le plus important en science est de retrouver cette description. L'épistémologie est une autorisation de parler et de se faire entendre. Mais déjà pour parler de réalité, il faut que cette dernière existe. C'est dans cette optique que deux paradigmes s'opposent :

1. Le positivisme ;

2. Le constructivisme.

Pour le projet positiviste, la connaissance se base sur l'observation et l'expérience des faits sociaux. Sous ce postulat, le travail de recherche se déroule selon un schéma linéaire et invariant :

Théorie $\rightarrow$ hypothèses $\rightarrow$ observations $\rightarrow$ généralisation $\rightarrow$ théorie

Le chercheur construit un système hypothétique- déductif avant de le confronter à des situations empiriques supposées représentatives. (Wacheux, 1996)

Quant au constructiviste, il coöncide avec une attitude ouverte de recherche, plutôt qu'avec un paradigme définitif. Le chercheur produit des explications, qui ne sont pas la réalité, mais construit sur une réalité susceptible de l'expliquer. L'épistémologie constructiviste abandonne la croyance d'un accès objectif possible au réel.

Etant donné la nature de notre recherche et nos croyances personnelles, nous adopterons provisoirement, au regard de notre statut, une combinaison des paradigmes positiviste et constructiviste. Donc, on se devait aussi d'être en même temps positiviste et constructiviste donc interprétativiste. Au regard du design de notre travail, nous pensons davantage nous rapprocher du paradigme positiviste concernant l'indépendance du chercheur vis-à-vis du terrain d'analyse, la prise en considération des faits observés avant tout, le test d'hypothèses.

Nous sommes proches du courant constructiviste pour ce qui est de la reconnaissance du rôle moteur des intérêts humains, de la tentative de compréhension des phénomènes dans leur globalité et de la reconnaissance du monde comme réalité subjective. En outre, le dépouillement de l'information ainsi que son traitement sera fait à l'aide du logiciel de statistiques SPSS.

\section{Résultats et Recommandations:-}

\section{Renseignements sur la Banque:-}

Les clients de votre banque sont-ils surtout des : (réponses multiples)

Les résultats ont montré que les PME sont les principaux clients des banques commerciales libanaises (94.70\%) suivis par les particuliers $(52.60 \%)$ et les grandes entreprises $(47.40 \%)$. Ces résultats mettent en évidence l'importance des PME comme marché pour les banques libanaises.

Quelle est la part de crédit accordée aux PME (en \%)

Tableau 4.1:-Durée des crédits accordés aux PME

\begin{tabular}{|c|c|c|c|}
\hline & à court terme & à moyen terme & à long terme \\
\hline Moyenne & 51.58 & 31.68 & 15.68 \\
\hline Mode & 60.00 & 30.00 & 10.00 \\
\hline
\end{tabular}




\begin{tabular}{|l|l|l|l|}
\hline Ecart type & 18.87 & 13.88 & 7.78 \\
\hline Minimum & 10.00 & 10.00 & 5.00 \\
\hline Maximum & 82.00 & 75.00 & 30.00 \\
\hline
\end{tabular}

Les résultats obtenus prouvent que les crédits accordés aux PME sont, dans la majorité des cas, des crédits à court terme. En deuxième position viennent les crédits à moyen terme tandis que ceux de long terme sont plus rarement octroyés à ce type d'entreprises.

\section{Les différents critères:-}

Pour l'étude d'un nouveau projet, quels sont les éléments à prendre en considération :

Tableau 4.2:-Eléments à prendre en considération pour l'étude d'un nouveau projet

\begin{tabular}{|c|c|c|c|c|c|}
\hline & Moyenne & Mode & ET & Min & Max \\
\hline Les critères financiers & 5.00 & 5.00 & 0.00 & 5.00 & 5.00 \\
\hline Les critères économiques & 4.53 & 5.00 & 0.51 & 4.00 & 5.00 \\
\hline La personnalité de l'entrepreneur & 3.53 & 4.00 & 1.17 & 1.00 & 5.00 \\
\hline
\end{tabular}

Les résultats montrent que parmi les éléments à prendre en considération pour l'étude d'un nouveau projet, les critères financiers viennent en premier lieu (moyenne $=5$ ) suivis par les critères économiques et ensuite par la personnalité de l'emprunteur.

Pour l'aspect financier du projet, classez les critères suivants:

Tableau 4.3:-Critères de l'aspect financier du projet à prendre en considération

\begin{tabular}{|c|c|c|c|c|c|}
\hline & Moyenne & Mode & ET & Min & Max \\
\hline Taille du projet & 3.16 & 4.00 & 1.21 & 1.00 & 5.00 \\
\hline Taux de rentabilité du projet & 4.84 & 5.00 & 0.37 & 4.00 & 5.00 \\
\hline Délais de récupération de projet & 3.95 & 5.00 & 1.18 & 2.00 & 5.00 \\
\hline Risque d'insolv. induit par le projet & 4.63 & 5.00 & 0.60 & 3.00 & 5.00 \\
\hline L'apport personnel & 4.00 & 5.00 & 1.11 & 1.00 & 5.00 \\
\hline Les garanties réelles & 4.74 & 5.00 & 0.56 & 3.00 & 5.00 \\
\hline Les garanties personnelles & 4.11 & 4.00 & 0.74 & 3.00 & 5.00 \\
\hline Le patrimoine du créateur & 4.37 & 5.00 & 0.76 & 3.00 & 5.00 \\
\hline Plan de financement & 4.53 & 5.00 & 0.61 & 3.00 & 5.00 \\
\hline
\end{tabular}

Les résultats figurant dans le tableau ci-dessus nous permettent de dire que le taux de rentabilité du projet est l'élément le plus important pour l'aspect financier du projet (La moyenne $=4.84$ ), les garanties réelles (La moyenne $=4.74)$, le risque de d'insolvabilité induit par le projet (La moyenne $=4.63)$ tandis que la taille du projet est l'élément le moins important (La moyenne $=3.16)$.

Pour l'aspect économique du projet, classez les éléments suivants :

Tableau 4.4:-Critères de l'aspect économique du projet à prendre en considération

\begin{tabular}{|c|c|c|c|c|c|}
\hline & Moyenne & Mode & ET & Min & Max \\
\hline Nature de l'activité: & & & & & \\
\hline Le produit & 4.47 & 5.00 & 0.70 & 3.00 & 5.00 \\
\hline Le marché et son évolution & 4.26 & 5.00 & 0.87 & 3.00 & 5.00 \\
\hline Etendu géographique du marché & 4.11 & 4.00 & 0.74 & 3.00 & 5.00 \\
\hline Position concurrentielle: & & & & & \\
\hline Part de marché espérée & 3.95 & 5.00 & 1.03 & 2.00 & 5.00 \\
\hline Le secteur d'activité & 4.16 & 5.00 & 1.01 & 2.00 & 5.00 \\
\hline
\end{tabular}

Les résultats figurant dans le tableau ci-dessus nous permettent de dire que, pour l'aspect économique du projet, le produit est l'élément le plus important en ce qui concerne la nature de l'activité (La moyenne $=4.47$ ), pour la position concurrentielle, le secteur de l'activité est l'élément le plus important (La moyenne $=4.16$ ).

Pour la personnalité de l'entrepreneur, classez les critères qui vous semblent importants : 
Tableau 4.5:-Critères de l'aspect de la pers. de l'entrepreneur à prendre en considération

\begin{tabular}{|c|c|c|c|c|c|}
\hline & Moyenne & Mode & ET & Min & Max \\
\hline L'expérience professionnelle & 4.32 & 4.00 & 0.48 & 4.00 & 5.00 \\
\hline Les qualités humaines & 3.95 & 5.00 & 1.18 & 2.00 & 5.00 \\
\hline Les motivations & 4.26 & 4.00 & 0.56 & 3.00 & 5.00 \\
\hline La connaissance du marché & 4.47 & 5.00 & 0.70 & 3.00 & 5.00 \\
\hline La connaissance du produit & 4.42 & 4.00 & 0.61 & 3.00 & 5.00 \\
\hline L'antécédent familial & 1.58 & 1.00 & 0.61 & 1.00 & 3.00 \\
\hline Niveau de formation & 3.95 & 5.00 & 1.03 & 2.00 & 5.00 \\
\hline
\end{tabular}

Les résultats figurant dans le tableau ci-dessus nous permettent de dire qu'au niveau de la personnalité de l'entrepreneur, la connaissance du marché est l'élément le plus important pour la personnalité de l'entrepreneur (moyenne $=4.47$ ) ainsi que l'antécédent familial est l'élément le moins important (moyenne $=1.58$ ).

Parmi les propositions suivantes, quelles sont les caractéristiques qu'un nouveau promoteur doit avoir :

Tableau 4.6:-Les caractéristiques qu'un nouveau promoteur doit avoir

\begin{tabular}{|c|c|c|c|c|c|}
\hline & Moyenne & Mode & ET & Min & Max \\
\hline Age & 4.26 & 5.00 & 1.05 & 2.00 & 5.00 \\
\hline Situation familiale & 2.63 & 3.00 & 0.96 & 1.00 & 4.00 \\
\hline L'environnement familial du créateur & 3.05 & 3.00 & 0.78 & 2.00 & 4.00 \\
\hline Qualifications et diplômes & 4.16 & 5.00 & 1.26 & 1.00 & 5.00 \\
\hline Activités professionnelles antérieurs & 4.26 & 5.00 & 0.99 & 1.00 & 5.00 \\
\hline
\end{tabular}

Les résultats obtenus montrent que l'âge et les activités professionnelles antérieurs sont les deux facteurs les plus importants qu'un nouveau promoteur doit les avoir (les deux moyennes $=4.26$ ), tandis que la situation familiale n'a pas une grande importance (moyenne $=2.63$ ).

Parmi les propositions suivantes quelles sont les motivations qu'un nouveau promoteur doit avoir :

Tableau 4.7:-Les motivations qu'un nouveau promoteur doit avoir

\begin{tabular}{|c|c|c|c|c|c|}
\hline & Moyenne & Mode & ET & Min & Max \\
\hline Goût d'entreprendre & 3.37 & 3.00 & 0.83 & 2.00 & 5.00 \\
\hline Volonté d'autonomie et d'indépendance & 3.21 & 3.00 & 0.54 & 2.00 & 4.00 \\
\hline Epanouissement & 4.58 & 5.00 & 0.69 & 3.00 & 5.00 \\
\hline Sens du relationnel & 3.95 & 5.00 & 1.18 & 2.00 & 5.00 \\
\hline
\end{tabular}

Les résultats obtenus montrent que l'épanouissement est la motivation la plus importante qu'un promoteur doit l'avoir $($ moyenne $=4.58)$ alors que la volonté d'autonomie et d'indépendance est la moins importante (moyenne $=$ $3.21)$.

Veuillez indiquer les qualités qu'un nouveau promoteur doit avoir:

Tableau 4.8:-Les qualités qu'un nouveau promoteur doit avoir

\begin{tabular}{|c|c|c|c|c|c|}
\hline & Moyenne & Mode & ET & Min & Max \\
\hline Prend des initiatives & 4.00 & 4.00 & 0.82 & 3.00 & 5.00 \\
\hline Prend des risques calculés & 3.84 & 4.00 & 0.90 & 2.00 & 5.00 \\
\hline Sens des affaires & 3.89 & 3.00 & 0.81 & 3.00 & 5.00 \\
\hline Contact avec les autres & 4.32 & 5.00 & 0.95 & 2.00 & 5.00 \\
\hline Sérieux & 4.74 & 5.00 & 0.65 & 3.00 & 5.00 \\
\hline Confiance & 4.47 & 5.00 & 0.84 & 3.00 & 5.00 \\
\hline Degrés de professionnalisme & 4.53 & 5.00 & 0.61 & 3.00 & 5.00 \\
\hline La maturité & 4.53 & 5.00 & 0.70 & 3.00 & 5.00 \\
\hline Bon manager & 4.32 & 5.00 & 0.82 & 3.00 & 5.00 \\
\hline Bon gestionnaire & 4.37 & 5.00 & 0.76 & 3.00 & 5.00 \\
\hline Honnête & 4.74 & 5.00 & 0.73 & 2.00 & 5.00 \\
\hline Dynamique & 4.00 & 4.00 & 0.67 & 3.00 & 5.00 \\
\hline
\end{tabular}


Les résultats obtenus montrent que le nouveau promoteur doit être surtout sérieux et honnête $($ moyenne $=4.74$ ) tandis que la prise de risques calculés vient en dernier place dans l'échelle des qualités du nouveau promoteur (moyenne $=3.84)$.

\section{La Collecte d'Information:-}

Face au manque d'information et l'absence d'historique, quelles sont les sources les plus utilisées :

Tableau 4.9 -Les sources d'information les plus utilisées

\begin{tabular}{|c|c|c|c|c|c|}
\hline & Moyenne & Mode & ET & Min & Max \\
\hline Documents comptables & 4.63 & 5.00 & 0.76 & 3.00 & 5.00 \\
\hline Etude de rentabilité financière du projet & 4.37 & 5.00 & 0.83 & 3.00 & 5.00 \\
\hline Consulter les fournisseurs, les clients ou autres banques & 4.63 & 5.00 & 0.68 & 3.00 & 5.00 \\
\hline
\end{tabular}

Les résultats obtenus prouvent que les documents comptables et la consultation des fournisseurs, des clients ou des autres banques sont les sources les plus utilisées lors du manque d'information et l'absence historique (les deux moyennes $=4.63$ ).

En moyenne, quelles est la fréquence des entretiens avec le nouveau promoteur

Tableau 4.10:-La fréquence des entretiens avec le nouveau promoteur

\begin{tabular}{|c|c|}
\hline Moyenne & 2.68 \\
\hline Mode & 3.00 \\
\hline Ecart type & 0.95 \\
\hline Minimum & 1.00 \\
\hline Maximum & 4.00 \\
\hline
\end{tabular}

Les résultats obtenus montrent qu'en moyenne, la banque fait trois entretiens avec le nouveau promoteur.

Quelle est la durée moyenne de l'entretien?

Les résultats obtenus montrent que $57.89 \%$ des banques trouvent que la durée moyenne des entretiens varie entre 30 minutes et 1 heure.

L'antécédent familial du promoteur influence t-il votre jugement ?

Les avis sur l'influence de l'antécédent familial du promoteur sur le jugement sont presque également répartis avec une petite différence en faveur de la réponse positive.

\section{La Perception du Banquier:-}

Dans le cas d'une nouvelle création, le critère relatif à la personnalité de l'entrepreneur, peut-il influencer la prise de décision?

Toutes les banques trouvent que le critère relatif à la personnalité de l'entrepreneur peut influencer la prise de décision dans le cas d'une nouvelle création.

Le montant demandé par le nouveau promoteur est-il souvent obtenu?

Les résultats obtenus montrent que le montant demandé par le nouveau promoteur est obtenu en $57.89 \%$ des cas.

Si non, le refus est dû à :

Tableau 4.11:-Les principales causes du refus

\begin{tabular}{|c|c|c|c|c|c|}
\hline & Moyenne & Mode & ET & Min & Max \\
\hline La situation de secteur d'activité & 3.20 & 4.00 & 4.40 & 4.40 & 3.20 \\
\hline L'insolvabilité de l'emprunteur & 3.00 & 4.00 & 5.00 & 4.00 & 3.00 \\
\hline L'insuffisance d'information & 0.84 & 0.71 & 0.89 & 0.55 & 0.84 \\
\hline Manque de garanties & 2.00 & 3.00 & 3.00 & 4.00 & 2.00 \\
\hline
\end{tabular}

Au cas où le montant demandé par le nouveau promoteur n'est pas obtenu, le refus est dû principalement à la situation du secteur d'activité ou de l'insolvabilité de l'emprunteur. 
Quels types de garanties utilisez-vous? (réponses multiples):-

Les résultats obtenus affirment que toutes les banques demandent des garanties réelles des clients et que $94.7 \%$ d'entre elles exigent des garanties sur le matériel de la firme (nantissement) et $89.50 \%$ demandent des garanties personnelles.

Dans quel cas exigez-vous toujours des garanties? (réponses multiples)

Tableau 4.12:-Cas exigeant toujours des garanties

\begin{tabular}{|c|c|}
\hline & Pourcentage \\
\hline Structure financière fragile & $84.20 \%$ \\
\hline Pour des montants importants & $84.20 \%$ \\
\hline Sur des affaires en création & $84.20 \%$ \\
\hline Pour tous types de crédits & $73.70 \%$ \\
\hline Pas de cas particulier & $42.10 \%$ \\
\hline
\end{tabular}

Les résultats obtenus montrent que la structure financière fragile, les montants importants et les affaires en création sont des cas qui exigent toujours des garanties ( $84.20 \%$ des cas) et que $73.70 \%$ des banques exigent toujours des garanties pour tous types de crédits.

Dans quel cas n'exigez-vous jamais des garanties? (réponses multiples)

Tableau 4.13:-Cas n'exigeant pas toujours des garanties

\begin{tabular}{|c|c|}
\hline & Pourcentage \\
\hline Structure financière fragile & $57.90 \%$ \\
\hline Pour des montants importants & $52.60 \%$ \\
\hline Sur des affaires en création & $52.60 \%$ \\
\hline Pas de cas particulier & $36.80 \%$ \\
\hline Autres & $36.80 \%$ \\
\hline Pour tous types de crédits & $26.30 \%$ \\
\hline
\end{tabular}

Les réponses à cette question montrent que la structure financière fragile, les montants importants et les affaires en création ne sont des cas qui exigent toujours des garanties (entre 50\% et 58\% des cas) et que $26.30 \%$ des banques n'exigent toujours des garanties pour tous types de crédits.

Par rapport à la garantie, l'étude de projet constitue une alternative:

Les réponses à cette question montrent que l'étude du projet constitue une alternative satisfaisante pour 57.89\% des cas.

Un client acceptant d'offrir beaucoup de garanties est : (réponses multiples)

La majorité des banques trouvent que les clients qui acceptent offrir beaucoup des garanties sont des bons clients.

Votre expérience affecte-t-elle vos décisions? Oui $=100.00 \%$

Comment jugez-vous, vos décisions prises durant vos années d'expériences?

Tableau 4.14:-Décisions prises durant les années d'expériences

\begin{tabular}{|c|c|}
\hline Moyenne & 4.74 \\
\hline Mode & 5.00 \\
\hline Ecart type & 0.45 \\
\hline Minimum & 4.00 \\
\hline Maximum & 5.00 \\
\hline
\end{tabular}

Sur 100 clients combien de projet sont acceptés?

Tableau 4.15:-Sur 100 clients combien de projet sont accepté

\begin{tabular}{|c|c|}
\hline Moyenne & 66.84 \\
\hline Mode & 65.00 \\
\hline Ecart type & 9.75 \\
\hline Minimum & 45.00 \\
\hline
\end{tabular}




\section{Maximum}

85.00

Ces projets sont-ils tous de bons projets?

Oui $=100.00 \%$

Comment jugez-vous, la satisfaction de votre clientèle quant aux services offerts par votre banque :

Les résultats montrent que les banques croient que leurs clients sont à $86 \%$ satisfaits de leurs services.

Avez-vous déjà suivi des cycles de formations en psychologie?

Les résultats montrent que $58 \%$ des répondants ont déjà suivi des cycles de formation en psychologie.

\section{Conclusions et Recommandations:-}

En réponse aux hypothèses, on met en avance ce qui suit :

H1- Le coût d'accès au financement est plus élevé pour les PME que pour les autres entreprises.

Cette hypothèse est acceptée parce que toutes les banques trouvent que les critères financiers sont les plus importants pour l'étude d'un nouveau projet, $94.74 \%$ des banques aussi trouvent que le plan de financement est un élément très important.

H2- Les PME «souffriraient» d'un déficit chronique de financement de longue période.

Cette hypothèse est acceptée parce que seulement $15.68 \%$ des crédits accordés aux PME sont des crédits à long terme.

H3- Les banques Libanaises sont incapables de cerner le risque présenté par la P.M.E

Cette hypothèse est acceptée parce que la majorité des banques ont évalué positivement la plupart des facteurs financiers, économiques, la personnalité de l'entrepreneur, les caractéristiques des clients, les motivations et les qualités qu'un nouveau promoteur doit les avoir.

H4- La surestimation du risque exige des primes trop élevées, les clients se sentent éliminés d'office.

Cette hypothèse est acceptée parce que les conditions que les banques trouvent qu'elles sont importantes sont nombreuses: taille du projet, taux de rentabilité, les critères financiers, les critères économiques, délais de récupération de projet, risque d'insolvabilité induit par le projet, l'apport personnel, les garanties réelles, les garanties personnelles, le patrimoine du créateur, plan de financement, l'expérience professionnelle, les qualités humaines, les motivations, la connaissance du marché, la connaissance du produit, niveau de formation, âge, qualifications et diplômes, et activités professionnelles antérieures.

H5- Les informations comptables produites par les PME libanaises sont très ambiguës.

Cette hypothèse est rejetée parce que la majorité des banques libanaises $(84.21 \%)$ trouvent que les documents comptables des PME sont les sources les plus utilisés dans le cas où il y'a manque d'information.

H6- L'attitude des banques à l'égard des P.M.E. est frileuse.

Cette hypothèse est rejetée parce que les banques ont acceptés $66.84 \%$ des projets (67/100 projets) en plus ils ont trouvé que les projets acceptés sont bons.

H7- Il existe une asymétrie d'information entre les propriétaires des PME et les banquiers.

Cette hypothèse est acceptée, parce que la majorité des banques demandent des garanties personnelles (89.50\%), en plus les banques trouvent que les propriétaires doivent être sérieux (89.74\%) et honnête (94.74\%).

Les recommandations sont adressées aux PME aussi bien qu'aux banques commerciales libanais. Concernant les PME, celles-ci sont appelés à adopter des politiques de gestion claires dans le but d'aboutir à une meilleure information financière concernant leur performance et activité qui leur serviront de preuves lorsqu'ils demandent des crédits auprès des institutions financières.

Quant aux banques, elles doivent opter pour une modification de leur politique d'octroi des crédits facilitant l'accès au financement surtout pour les PME qui forment la majorité des entreprises libanaises, surtout que celles-ci imposent des garanties importantes lors de l'octroi de ces crédits. 


\section{Références:-}

1. Bardos M. (1991), la liaison entre le risque et la taille : influence du risque et de la taille sur le taux d'interet, cahiers economiques et monetaires, n38. Pp.49-85.

2. Gambetta, D. (1988), can we trust trust ? in trust, making and breaking cooperative relations. Oxford Basil Blackwell.

3. Hugon, Ph. (1990), La finance informelle : expression de la crise du développement ou de nouvelles formes de développement ? in Lelart (M) (éd) pp. 309-321

4. Keynes, J.M. (1921), a treatise on probability, the collected writtings of John Maynar Keynes, vol. VIII, Macmillan, 1973.

5. Wacheux, F. (1996). Méthodes qualitatives et recherche en gestion. Economica.

6. Thietart, R. A. (2003). Doing Management Research: A Comprehensive Guide. JOURNAL-OPERATIONAL RESEARCH SOCIETY, 54(10), 1123-1123.

7. Quinet, C. (1994). Herbert Simon et la rationalité. Revue française d'économie, 9(1), 133-181.

8. Simon, H. A. (1982). Models of bounded rationality: behavioral economics and business organization, vol.. The Massachusetts Institute of Technology. 